\title{
Mediating identities: Sign language interpreter perceptions on trust and representation
}

\author{
Jemina Napier, Robert Skinner, Alys Young and Rosemary Oram
}

\begin{abstract}
Deaf people's lives are frequently predicated on working with interpreters. Identity becomes known and performed through the translated self in many interactions with hearing, non-signing people. Taking an interdisciplinary approach in combining interpreting studies, deaf studies, applied linguistics and social research, the 'Translating the Deaf Self' project, funded by the Arts and Humanities Research Council (UK), sought to explore the experience of deaf people and other stakeholders of the lived experience of being translated. Drawing on discourses of identity, representation and trust, this paper gives an overview of the findings from two focus groups with sign language interpreters $(n=7)$ on their perspectives of the experiences of deaf signers being 'known' through interpreting. Social constructionism underpinned our approach to data analysis and the dominant theme of 'trust' was examined with reference to a framework for trustworthiness developed by Alan Jones and Samantha Sin. In particular, we focus on the issue of trust in relation to representation, relationships, ability and boundaries. The main findings demonstrate that sign language interpreters are acutely aware of the responsibility they have to represent deaf signers, especially at work, and thus represent their professional-and-deaf identities, and the important role of trust for deaf professionals to feel represented through interpreters.
\end{abstract}

KEYWORDS: DEAF SIGNERS; MEDIATION; PROFESSIONAL IDENTITY; REPRESENTATION; SIGN LANGUAGE INTERPRETERS; TRUST

\footnotetext{
Contact author

Jemina Napier: Centre for Translation \& Interpreting Studies in Scotland, Department of Languages and Intercultural Studies, School of Social Sciences, Henry Prais Building, Heriot-Watt University, Edinburgh, EH14 4AS, Scotland, UK.

Email: j.napier@hw.ac.uk
} 


\section{Introduction}

Sign language interpreting as a professional practice is situated within the wider disciplines of applied sign linguistics and interpreting studies, and sign language interpreters (SLIs) engage in communicative mediation between deaf and hearing interlocutors in a range of contexts (Napier and Leeson 2016). Studies that have explored the relationship between SLIs and deaf people (e.g. Napier and Rohan 2007; De Wit and Sluis 2014; Haug et al. 2017) have not considered in depth the issue of representation: the fact that SLIs not only have to mediate communication, but that deaf signers are largely 'known' through the SLIs, particularly when the interpreter is (literally) the 'voice' of the deaf person.

Representation is both ontological (how the other might be present to us) and epistemological (how another's point of view and knowledge is conveyed). SLIs can be agents of representation in both of these senses. Therefore, the extent to which SLIs actively engage in processes of the social construction of the deaf self - through, for example, their lexical choices, their tone of voice, the choice of register in representing the deaf person - is of interest.

The 'translated deaf self' (Napier et al. 2019; Young et al. 2019; Young et al. in press) is a regular feature of everyday experience. Although deaf signers are typically bilingual to some extent (in for example British Sign Language and spoken and/or written English), and engage in multilingual practices (De Meulder 2016), their linguistic relationship with the world is often predicated on working with SLIs. Crucially, identity becomes both known and performed through the translated self in many interactions.

The 'Translating the Deaf Self' project ${ }^{1}$ sought to explore deaf identities and representation through the perspectives of deaf signers, hearing colleagues of deaf people and SLIs, to examine how translation/interpreting may be constitutive of deaf culture(s) in their formation, projection and transformation, and the nature of the impact of consistently experiencing existence to others as a translated (interpreted) self on personal identity, achievement and wellbeing.

Drawing on discourses of identity and trust from social identity theory and applied linguistics, this paper details focus group discussions with SLIs and offers a new theoretical perspective on the relationship between SLIs and deaf signers in relation to the notions of representation and trust. The focal point of analysis is the extent to which SLIs recognise that they represent, and therefore to some extent co-construct, deaf identities, and the central role of trust in ensuring representation. Our specific research questions for this sub-study are:

1. Do SLIs recognise their responsibility in mediating deaf-and-professional identities?;

2. What role does trust have in enabling SLIs to represent deaf-andprofessional identities?; and 
3. What do SLIs feel are the necessary components of their working relationship with deaf service users in order to mediate language, culture and identities?

This paper initially provides an overview of the relevant literature in order to contextualize the study, giving consideration to the key concepts of identities, interpreter-mediation, representation and trust, before describing the methodology used in arranging, conducting and analysing semi-structured interviews. We then report our findings, discussing notions of representation and trustworthiness, before acknowledging the limitations of the study and concluding with recommendations for further research.

\section{Literature review}

\subsection{Identities, interpreter-mediation, representation}

From a social identity theory perspective (Joseph 2004), identity is dynamic and constantly evolving, context dependent (Bucholtz and Hall 2004) and (to some degree) a learning process (De Clerck 2017). So, we enact and present alternate identities in different contexts, and identities are perceived, in part, through our linguistic and communicative choices (Marra and Angouri 2011; Howarth 2016). Professional identity is a self-concept based on attributes, beliefs, values, motives and experiences (Ibarra 1999), and professional roles and identities are performed at work on a daily basis (Sarangi 2010; Marra and Angouri 2011). Social interaction presupposes the existence of relatively stable identity types as a response to 'the need for legitimating one's presence while remaining other-oriented' (Sarangi 2010: 30). Thus professional identity is actively constructed, but only partly stable, and perceptions of professional identity validate role performance (Sarangi 2010).

Slay and Smith (2011) suggest that although professional roles are typically defined as prestigious and confer the role holder with varying degrees of autonomy, stigmatised persons are not accorded the same level of prestige and/ or privilege, because their identities could be perceived as tainted. Thus, deaf professionals need to ensure that their professional identities are not compromised by the potential 'stigma' of being deaf, so mediation and representation of their professional rather than deaf identity, or their 'deaf-and-professional identities', is crucial in order to succeed in the workplace (Napier et al. 2019).

If, however, one's identity is mediated through another person, i.e. an interpreter, and that interpreter makes linguistic choices in a different language on your behalf, it is their linguistic choices that impact on others' perceptions of their identity. This situation is one that is the lived experience of deaf signers, particularly in professional settings. 
Various backgrounds lead deaf people to deaf communities and sign language use, so linguistic and cultural diversity is evident within signing communities; and deaf people may have more than one identity depending on the context in which they are interacting with others (Bat-Chava 2000; Leigh 2009). Increasing provision of SLIs in universities has led to more deaf people gaining professional qualifications and employment (Boutin and Wilson 2009) and thus increasing presence of SLIs in professional workplaces (Dickinson 2014; Miner 2017).

Feyne $(2015,2018)$ has delved deeper into the representation of identity through SLIs by examining museum tour guide trainers' evaluations of interpreted gallery talks delivered by deaf museum guides. The deaf guides were well established in their practice and had experience in delivering museum talks in sign language to deaf members of the public. All of the evaluators were hearing, non-signing, museum trainers, so they could only judge the deaf guides' abilities based on the quality of the spoken English interpretation. Feyne found a misalignment between an understanding of the interpreter's task and the recipient's assumptions about the way SLIs function. Essentially, Feyne suggests that, in line with positioning theory (Van Langenhove and Harré 1994), no matter how much a person tries to convey their own identity, it is the recipient of the message who assigns identity. Our study brings a new perspective by considering the perceptions that SLIs have about how well they can represent the deaf signers with whom they work. In particular, we make a contribution by considering the relationship between representation and trust.

\subsection{Trust}

Trust is socially constructed and accomplished through everyday interaction (Gillespie 2008). It is more than an expectation; it is relational (Markova et al. 2008). Actions, including speech acts, produce trust, and once produced, trust becomes a fact of the relationship as it makes people feel safe, and therefore influences future actions. So, trust structures interaction and is intimately connected to the self (Weber and Carter 2003). A relational - communicative, rather than behavioural - view of trust is that it is a process, and that people mitigate the potential violation of trust through having faith that trust is reciprocal.

As such, trust-related and trust-bearing issues are 'central to our understanding of how the conduct of professional practices impacts on human relationships in social life' (Candlin and Crichton 2013a: 1). Further, '[w] hen we say that we trust someone or that someone is trustworthy, we implicitly mean that the probability that he will perform an action that is beneficial or at least not detrimental to us is high enough for us to consider engaging in some form of cooperation with him' (Gambetta 1988: 217). Thus for SLIs working with 
deaf professionals, trust relates to whether the deaf professional trusts that the interpreter performs the action of interpreting that is beneficial to their professional interactions (Napier and Leeson 2016).

The relationship between those who trust and are trusted raises questions of how trust is to be guaranteed and what ontological status to ascribe to trustors and trustees (Candlin and Crichton 2013b). Essentially, trust is associated with the behaviour of others; perception of the trustworthiness of others is associated with ontological security and mutual trust is a condition of cooperative behaviour. Trust is socially and discursively constructed, which as a practice manifests as a form and condition of social action. Mayer et al. (1995) suggest a model for examining trust-related issues in professional (and organisational) contexts based on clients' perceptions of the ability, integrity and empathy of the person that is being trusted (the trustee); that is, their trustworthiness. For the purposes of this paper, then, the client is the deaf professional and the trustee is the interpreter; and our interest is in how SLIs perceive that they are trusted by deaf professionals.

Jackson (2013) observes that mutually beneficial relationships rely on people making positive evaluations of each other's' actions and interactions, which then lead to the development of confidence and trust in one another - i.e., interpersonal trust. Developing such trust is a time-consuming process requiring commitment, but in the case of deaf professionals and SLIs, if the relationship works then the deaf professional will get to interact with hearing people at work, and the interpreter will get offered more work.

Sin and Jones (2013) define workplace trust as trusting that a service will be delivered. In relation to professional trustworthiness and identity work, they found that accountants are trusted to add value to a firm's bottom line, but are simultaneously trusted to adhere to the high ethical standards of their profession while complying with stringent government regulations. Accountants adopt a range of communicative functions, which are key to successful practice and allow them to cope with the competing expectations and obligations of their role while constructing and maintaining a trustworthy persona. Sin and Jones's model can be applied to interpreting, in that a deaf individual trusts that the SLI will turn up, conduct themselves ethically and professionally and interpret accurately. This could be considered as a form of 'anticipatory trust'; that is, trust as a prerequisite rather than trust enacted through interaction of trust gained through experiences. However, we are not interested in the service provision alone. Rather, we here also focus on whether SLIs feel that deaf professionals trust them to represent their professional identity.

Sin and Jones's interviewees identified a number of 'inferred attributes/ dispositions' that they refer to as 'indexes of trustworthiness'; the terms used 
include (1) being accurate/correct (the authors interpret this as being ethical), (2) giving and deserving respect, (3) being competent (having expert abilities), (4) being benevolent and (5) being in control. All of these attributes are also relevant to the trustworthiness of interpreters. In the case of accountants, Jones and $\mathrm{Sin}$ suggest that communicative expertise in particular is needed to establish trust. However, while this requirement can also be applied to interpreting, as communication is at the heart of what they do, a more important factor may be how they empower the deaf professional and how deaf professionals perceive they are viewed through the communicative expertise of the interpreter.

Although an under-researched area, it has been found that minority-language service users perceive trust as a critical component in relationships with interpreters (Edwards et al. 2005; Edwards et al. 2006; Napier and Rohan 2007; Hsieh et al. 2010; Tipton 2010; Napier 2011; Holcomb 2018), and that it has a direct bearing on their comfort with particular interpreters and confidence in the interpreting process. Also, Edwards (2013) has discussed the role of interpreters in enabling her to do her job as a researcher when interviewing minority-language speakers, and identified trust as a critical element in preventing a sense of powerlessness in communicating her needs in her role. Yet no prior work has given in-depth consideration to issues of representation and trust in relation to the interpreter-mediated professional identity of deaf signers and the perception of SLIs on the role of trust in communicating the needs of deaf professionals. Thus we were interested in examining whether SLIs recognised their responsibility in mediating deaf-and-professional identities, the role of trust in enabling SLIs to represent these identities and what SLIs felt were the necessary components of their working relationship with deaf service users in order to mediate language, culture and identities.

\section{Method}

A social constructionist ontology underpins this exploratory study. We acknowledge that both experience and interaction construct and co-construct realities, that meaning is inter-socially produced, and that diverse social discourses occupy varying degrees of authority in influencing meaning production (Burr 2015). Thus we declare that Napier and Skinner, who were the primary data collectors and led on the analysis of this data, are both sign language interpreters and that they bring their lived experiences as interpreters to the study. Young, who contributed to the interpretation of the data and its presentation, is hearing, has worked alongside deaf people as a sign language user for nearly 30 years and with Napier was the originator of the project and the concept of the translated deaf self. Oram is deaf with the lived experience of 
being regularly interpreted in professional and non-professional roles. Ethics approval was received from both the University of Manchester and HeriotWatt University School of Social Sciences Research Ethics Committees.

\subsection{Participants and process}

Participants were recruited using network sampling: that is, via personal contacts, professional interpreter distribution lists and e-list groups and social media. The only selection criteria were that respondents had to be qualified SLIs. Individuals who responded were provided with information about the date, time and venue of focus groups.

Seven SLIs participated in two focus groups in Scotland $(n=4)$ and England $(n=3)$. The sample included five females and two males, and were aged 34 to 59. Four participants have deaf family members; two learned BSL before the age of 12, and five learned BSL as a second language in their late teens or early to mid-twenties. Three are employed by an interpreting agency and the remaining four all work as freelance SLIs. All participants have highereducation qualifications in interpreting studies and regularly work with deaf professionals.

The seven participants were provided with the questions to consider in advance. Each focus group lasted 1.5-2 hours and were both carried out by Napier and Skinner, in order to 'match identities' of researchers and participants. We believe this peer approach to data collection encouraged a deeper exchange of views because the participants knew that the researchers would understand from both professional practitioner and researcher perspectives.

\subsection{Analytical procedure}

The focus groups were conducted in spoken English but included the spontaneous use of signed expression. The groups were video-recorded and afterwards transcribed and anonymised. The first stage of thematic analysis involved generic coding for key themes, and was co-analysed by Napier and Skinner using N-Vivo qualitative analysis software. ${ }^{2}$ Through our thematic review of the data, 'trust' emerged as a recurring theme. To better understand this theme we reviewed the applied linguistics literature for existing trust analysis frameworks, which led us to Sin and Jones's (2013) indexes of trustworthiness as discussed above, which we then used to interrogate the data further, looking for collocations of frequently used terms in relation to trust. This led to the identification of four sub-themes: (1) representation, (2) relationships, (3) ability and (4) boundaries. Table 1 shows how these themes relate to the index terms identified by Sin and Jones. The data was then re-analysed in relation to these indexes, and checked and confirmed by other members of the research team. 
Table 1: Analysis themes

\begin{tabular}{|l|l|l|}
\hline Sin and Jones' indexes of trustworthiness & Our sub-themes & \multirow{2}{*}{ Overall theme } \\
\hline Give and deserve respect & Trust and representation & \\
\cline { 1 - 2 } Benevolent & Trust and relationships & \\
\cline { 1 - 2 } Competent (expert abilities) & \multirow{2}{*}{ Trust and ability } & \multirow{2}{*}{ Representation } \\
\cline { 1 - 2 } Accurate, correct (ethical) & \multirow{2}{*}{ Trust and boundaries } & \\
\cline { 1 - 2 } In control & & \\
\cline { 1 - 2 } & &
\end{tabular}

\section{Findings: Notions of representation and trustworthiness}

The initial focus of the analysis is on how SLIs feel they can best represent deaf signers and their professional identities and how they understand the concept of 'representation'. It became clear, however, that trust and relationships, ability and boundaries are all central to whether representation can be achieved. Thus we begin by discussing the role of trust in relation to representation, followed by the other categories.

\subsection{Trust and representation}

Both focus groups discussed why trust was an important part of enabling representation. The need to develop trust was not just considered in terms of being sensitive towards someone's privacy and using that trust as a symbol of confidentiality. The SLIs noted that deaf signers have to trust the interpreters to represent them, but also trust that the SLI understands how they want to be represented, as explained by Heidi:

I think not only trusting that you will represent them well but also trusting that you understand how they want you to do it. Sometimes you kind of have to know what they want to portray.

This was also highlighted by Linda, who discussed how she had not represented a deaf person's mood correctly, and how it had impacted on the interaction:

I think a lot of time it's just through making a lot of mistakes so actually having an angry voice when this deaf person looks like they are angry where you could have maybe slightly softened it, and it's only through those mistakes and getting yourself in a situation that you are like oh my God, what the hell happened there? I never want that to happen again. Then, when you sort of reflect back on it, you think if I had of done that it would have went a bit better.

All of the SLIs noted a tension between representing the deaf person's identity and ensuring one's own identity as the interpreter did not interfere. An example was given by Karen: 
I'm always very concerned about me bleeding through. I remember doing a court case, which was about a fight in a pub... The deaf guy was incredibly clear about what had happened in the corner of this pub, he was quite expressive and I chose to use the word 'fracas', which wasn't him at all; that was me. I think they must have gone, 'Oh, it was all this fracas!' which is funny but it's not because they are not being represented properly. It may be that if I'd represented him properly there might have been different ways that he was treated.

What Karen describes is the fact that SLIs have to make decisions concerning how to articulate the utterance of a person into another language, and that they ultimately make subjective choices about how to do that. What is interesting is that the choices made by the interpreter, whether lexical or not, can potentially portray the deaf person in a different light. The representation of that deaf person might be skewed, who consequently might not be 'seen' in the same way. SLIs are confronted with a choice: do I interpret that utterance in a way that I would say it, or how I think s/he would say it? That is, how much of my own self (as an interpreter) do I let 'bleed through'? And if one does not know the deaf person well, how easy is it to make that choice? Based on the stance taken by this interpreter, it would seem that the interpreting product is wholly dependent on conscious decisions made by the interpreter about how they represent ideas/events/people. Implicit within that decision-making is the trust from deaf signers that SLIs make appropriate choices to portray their identity - a task which cannot be easy, as noted by Sharon:

It's so important that you always remember you are... representing the deaf person and their professional capabilities and their character and their personality. Not that you're responsible for it, but that you feel this responsibility for portraying it in some way, although people can obviously see a deaf person and see how they are and make their own mind up in that respect. In terms of their communication skills and how they present themselves through their voice, it's such a responsibility to do that and some deaf people are more trusting than perhaps they should be?

As Sharon states, both the interlocutors can see each other and formulate opinions of each other through other means. The interpretation produces many information streams that are used to formulate an understanding and impression of one another. Seemingly, our participants were aware that not only were they 'auditioned' prior to being selected for an assignment, but they were also being auditioned throughout an assignment. The notion of 'monitoring' the work of SLIs is a key component in how deaf signers assess how they are being represented. Sharon continues:

Because deaf people have to trust that you're representing them as they wish to be represented. Trust from the deaf person, from your point of view, that they trust that you will do that and you can see it in the way they respond to you that they like the way that you're 
signing, they understand the way you're signing. So they then trust that you will voiceover for them in a way that represents them well, even though they might not be able to monitor that... so I think trust is a very big part of that relationship.

We also consider representation in the other direction: how hearing interlocutors are represented to the deaf person through the interpreter. In our data we found examples of mediation by SLIs to support the deaf person to gain a better understanding of the context and/or to represent him/herself better in the interaction, especially when there are power asymmetries - for example, by providing information on how a hearing person is saying something so that the deaf person can respond to in the appropriate way. As noted by Heidi:

One thing I have noticed is my one day a week doing [video remote interpreting] is the fact that a lot of that information about the hearing person and what they might be doing, the tone of their voice, because I think sometimes deaf people can see for themselves how it's going. They can see from the way the hearing person is reacting, how they're reacting to you, but when you just have a face and a voice that awareness is actually expanded because I'm now having to say, 'Just to let you know, they sound really, really unhappy'. 'They sound in a rush', or it's all the extra things that you have to add on top that they're not able to see for themselves.

This example given by Heidi captures the importance of deaf signers needing to trust that representation is occurring in both directions, in order that the deaf and hearing interlocutors can exchange information on an equal footing.

\subsection{Trust and relationships}

The sub-theme of trust and relationships is concerned with how the feeling or development of trust between individuals can contribute to better relationships, and therefore representation, either in a personal capacity that later benefits the interpreted interaction, or solely in a work context. For SLIs this means 'if we fit well together and understand each other, trust will emerge between us', or alternatively, 'if we have trust between us this will enable us to understand each other better. The opposite can also occur, though, in that 'if the relationship between us does not improve, trust may diminish or fail to grow'.

The central notion of 'trust' emerged out of discussions about what constitutes a good relationship between interpreters and deaf signers. The two concepts of trust and relationships were interconnected in various ways by different interpreters, but all agreed that it was foundational to effective practice. Martin remarked:

You have got to develop trust with the communities. It's probably the strongest part of an interpreter's identity isn't it with the community? If you've got that level of trust, then all else is possible. If you have got genuine trust between your clients and yourself then you can do a lot more as an interpreter because they will trust you. 
One of the ways in which trust was built up was through the development and maintenance of good networks within deaf communities. Networks are important because they are the mechanism for developing positive relationships, which in turn are seen as essential to trust, as noted by Sharon:

[There are] people that I work with, quite a lot of people who I've known for a long time, so I feel that that's a very positive relationship. We work very well together. A lot of trust has been built up between us and I think that's important. Then of course when you meet new deaf people, it's also a challenge hoping that they can immediately trust you to do a good job for them and [have] some mutual respect and mutual liking...

SLIs felt that the character of their networks reflected their own individual characteristics and styles in relationships: some were more visible than others, some smaller than others, and they were considered an important element in establishing and maintaining trust, as noted by James:

For trust to exist you have to have a relationship with deaf people outside of the interpreting relationship. Now, however that takes form, but it's obviously easier for people who have got a deaffamily for that to happen on a semi-regular or regular basis.

However, Karen, who has deaf parents, felt differently:

I probably have a better connection with deaf people when I'm working as an interpreter professionally than I do out in the community in the social world, because I'm naturally quite shy. I find that I avoid that. So my relationship with communities is very professional. Of course I have deaf friends but that's one-to-ones and I quite often observe other interpreters who are very entrenched in the community. They often go to big deaf events and I think should I be doing that? I don't know. I'm confused! I'm doing a good job professionally, but do I need to be seen more out and about? So I think in terms of that trust... I think sometimes there's a sense that you are trusted more if you are seen out doing things with deaf people, not just in that working space.

So, although the close proximity (including the frequency of contact) between deaf signers and SLIs was an important consideration, and was seen to influence how relationships are formed, trust and relationships are not therefore automatic just because of growing up in a deaf community. It is common for a deaf person to see the same interpreter in different contexts, and also cross paths in social, non-interpreted, community contexts. James gave the following example:

I'm amazed how magnanimously deaf people accept us because I mean we're there on a Monday when they're getting a disciplinary at work, we're there on a Tuesday when the doctor is giving them cream for their piles, we're there for their couples' counselling because their marriage is falling to bits on a Wednesday and then we see them on Saturday at the theatre and they are delighted to see us! I wouldn't be.

This frequency of contact, in interpreted and non-interpreted contexts, means deaf individuals and SLIs both have to carefully consider how to maintain 
good relations and formulate a type of trust that applies to both interpreted and non-interpreted encounters. For deaf people the risk is higher because they are relying on SLIs, and exposing their lives to them, as James noted:

I've spoken to a deaf person who's had interpreters in very difficult situations who were great interpreters, really good interpreters, but she said, 'I wouldn't use them in everyday situations because they were there at a difficult time in my life. I don't want them there for my kids' parent's evenings', and it's no criticism of the interpreter, they were wholly professional and skilled, but just I would choose not to have them.

This example highlights the fact that trust is formed by visible exposure of the self in multiple contexts; therefore, if one is seen out of professional role this means that their behaviour out of that role is also a component of how trust is built, because it is visible too. This wish to not mix different parts of a person's life is no different to a patient not wanting to see their counsellor at a dinner party, but deaf signers and SLIs experience simultaneity of roles and therefore relationships, and so multiple contexts need to be held in balance, and trust is an integral part of that. The example from James also relates back to another facet of trust and working relationships, which is that trust has to be mutual, which was expressed as such by Heidi:

It's really important - a working relationship, so I really appreciate it when I feel like somebody is working with me instead of just throwing a bunch of stuff out and thinking I'm a machine. They can just turn me on and I can just get everything... So I see it more as a working relationship and I think trust is important, but I think that also it really needs to be a two-way street.

Doing a good job technically - that is, having the ability to interpret - is also the basis of trust, and therefore a deaf person feeling represented.

\subsection{Trust and ability}

All of the SLIs acknowledged that deaf people need to be able to trust that interpreters have the ability to do the job and to do it well. Karen recounted a situation where SLIs felt that they did not have the ability to represent the deaf person accurately, leading to tension:

I did a conference recently for somebody where I really felt that I struggled so much to represent that person exactly as they were that I'd let them down. I wasn't doing a poor job, I just wasn't able to find the words that were them [speaker's emphasis] and I felt disappointed, and I had worked for them a lot that day. It wasn't working. I felt a great sense of responsibility because they were in a public space and they were being listened to by lots of people and I let them down.

Needing to have competence is something that the SLIs reported being cognisant of, and Jen gave a specific example of how she tried to extend her skills to 
be better able to interpret more generally, but did not have the ability to work in that specific context:

I have situations that in order to stretch myself I'll put myself into [for example]... I do not want to do theatre. It's not something that interests me, and I think because of that I would panic and drown, so I've put myself in a situation where I've gone along and I've volunteered... because I thought right, okay, that's standing on stage, I have to learn how to [do that]... standing on the stage doing a little bit of theatre interpreting, and that just put the nail on the head, that is not an area that I want to touch. I know that now, but you've got to do that to know that.

One of the salient points that emerged in relation to trust and ability was that the SLIs expected to develop their competence over a period of time, and they were thankful to deaf people for supporting them on that journey. They recognised that without the trust and support from deaf communities, they might not reach the required level of expertise, as James described:

When you're a guest in someone's house, you are not part of the family, but you're made to feel very welcome. You are made to feel a part of things... I thought it was a great way of describing our relationship with deaf people, as guests in the community... when I started as a trainee... I didn't have the language skills that I really needed to be an interpreter... and the deaf community were so kind and so encouraging... I feel that I owe them so much for their patience and support and kindness in helping me to get confidence...

\subsection{Trust and boundaries}

Interpreters are bound by a Code of Conduct, which requires them to retain confidentiality, act impartially and to interpret accurately and faithfully. Therefore, there are clear boundaries of what should be expected of an interpreter in their professional role, and part of that role is to take responsibility to represent people to others, and not to insert their own self over and above the identities of others. Interpreters are participants in the interaction and coconstructors of meaning, so our understanding of role boundaries has moved on from rigid notions of invisibility and neutrality, as evidenced by Martin's comment:

We're there for communication and if our little bit of extra work helps the communication to move better... And so it's making ourselves visible to make it almost like we weren't there, if that makes any kind of sense... Because before we've tried to be invisible and just let stuff happen, and now we're more active participants so the result in the end as if we hadn't been there.

The crux of Martin's comment is that interpreters, in giving recognition to their own presence, rather than trying to pretend they are not there, they are more likely to blend in and thus enable communication to happen. An implication of Martin's comment is that boundaries are understood as being more 
fluid, and that allowing interpreters to manage communication (do that 'little bit of extra work') means that interlocutors trust that interpreters perform within the boundaries of their role appropriately. What becomes particularly awkward is when hearing people do not understand the boundaries of the interpreter, in that although the interpreter can be active in interpreting interactions, they are not there in their own right - they are only present to mediate between deaf signers and others. Sharon reported how she found it difficult when people addressed her directly:

One thing I sometimes find difficult to deal with is that fascination [that] extends to the interpreter. They're fascinated by what you do. 'How do you do that? Wow, that's amazing. You did a great job! That's amazing that you know!' A lot of times those questions, because they can communicate directly with you, a lot of those questions and those fascinations get directed towards you as the interpreter when really the focus shouldn't be on the interpreter. So that's always difficult to navigate in a polite way, but also try and re-shift the focus where necessary.

Sharon then noted that, in order to establish trust with deaf people in relation to boundaries, her response needed to be explicitly agreed:

I do work with some deaf people who I discuss that with: how do you want me to operate if people come and ask me questions? Some say, 'Oh, that's fine.' They know that I'll say the right thing, I hope! So again, it goes back to that trust thing, but you don't want it to happen too much because then [hearing] people become too used to talking to you.

The underlying issue is how one's behaviour as an interpreter can be regarded as a reflection of the identity of a deaf person. In the example above Sharon is aware of her boundaries and agrees with the deaf person on where and when it is appropriate to be a visible participant. The key is that the deaf person allows Sharon to have a presence, for her self to be seen, by allowing her to respond to a hearing person who may try to engage directly with her in a side conversation. The deaf person is secure enough in their own identity for that to happen. In relation to the notion of representation, it may be that the deaf person feels that Sharon is an extension of their own identity, and as Sharon herself notes, the deaf person trusts her to 'say the right thing. The deaf participant shows signs of trust and has granted the interpreter some leverage with how and when she can interact with other hearing participants. James provides a similar example, but does not consult; he makes the active decision to establish clear boundaries and reduce his presence by shifting the focus to the identities of deaf people:

And the one [ethical tenet] that I thought about was impartiality strangely, not because I want to be impartial, but it's about empowerment. I don't want to be taking control of a situation that doesn't belong to me. I don't want people looking at me. I don't want to be taking control, my voice. For me it's all about the people that are communicating because 
of the history of deaf oppression, and paternalism and the missioners and for me that's the most important thing to empower other people.

\section{Discussion and conclusion}

It becomes clear from our analysis that trust is a significant component in ensuring the representation of deaf-and-professional identities. The interpreters in this study were highly cognisant of the fact that deaf-and-professional identities were known, represented and co-constructed through interpretation.

In terms of trustworthiness, the different examples we have discussed illustrate that trust is developed through SLIs making it clear that deaf signers are (or at least should be) in control of how they are being represented. The interpreters in this study identified that minimising their potential for a discordant impact on the representation of deaf people's professional identity was as important as doing a good job technically. We suggest that these considerations relate to Sin and Jones's (2013) category of benevolence on the trustworthiness index. We have highlighted examples that illustrate how SLIs are well-meaning towards their clients in trying to do their best for them, in focusing on developing relationships and therefore trust.

There is a clear link between our categories of 'trust and relationship', and 'trust and ability', in that there is benevolence from deaf communities towards SLIs in order to support them in developing their competencies and expert abilities, which Sin and Jones (2013) also identify as a measure of trustworthiness. If SLIs display competence in terms of technical interpreting skills, provision of accurate interpreting and ethical behaviour, deaf signers are more likely to trust that the SLIs will also have the ability to represent them when they interact with non-deaf people. Trusting SLIs' ability has two dimensions: in addition to trusting their competence to make linguistic decisions, there is also a need to trust their professional decisions, and whether they know, understand and perform within the boundaries of the role of the interpreter.

Boundaries are also an aspect of effective representation in that if an interpreter transgresses these it can problematise the extent to which their

representative function is recognised and effective. To non-signing hearing people not used to working with interpreters, stepping out of role boundaries can be confusing, thus affecting perceptions of the identity of the deaf person. Our data reveal that SLIs have some control over boundaries so that they can do their job accurately, correctly and ethically, in alignment with the elements identified by Sin and Jones (2013); but rather than the trust coming from the client to interpreter in trusting that the interpreter is in control, it is the deaf client who trusts the interpreter to let them lead, and that the interpreter will make it clear that the deaf person is in the lead, so that there is a balance 
of control. Our data also reveal that there are many channels to gaining an impression of the other, but that not all are equal or are afforded equal status the voice and the spoken word remain asymmetrically powerful (Batterbury et al. 2007; Young et al. 2019), and interpreters are acutely aware of this.

In applying the indexes for trustworthiness identified by Sin and Jones (2013), it can be seen that SLIs feel that, essentially, deaf people deserve respect in their identities, that SLIs can give respect to deaf signers by thinking carefully about how to represent them and that deaf signers can in turn give respect to SLIs by trusting them. One way to ensure that trust of representation is possible is through the development and maintenance of relationships.

In revisiting the specific research questions, the data also confirm that SLIs recognise their responsibility in mediating deaf-and-professional identities, that they feel that good relationships are important in representing deaf signers and that trust has an essential role in their working relationship with deaf signers in order to mediate language, culture and identity.

The SLIs in our study are cognisant of the impact on both deaf and hearing people in not knowing each other: deaf signers rely on SLIs to represent them and give them voice, but SLIs also need to represent hearing people to deaf people as part of the process of mediation between two languages and cultures. However, they feel more of a responsibility towards making sure that deaf signers are known, as they perceive that deaf people experience so many more barriers, and trust is an integral part of the relationship to ensure that representation is achieved. Thus it could be argued that the deaf cultural experience, in the eyes of interpreters, is comprised of a state of being that incorporates a translated self.

In conclusion, this study has provided an exploration of seven sign language interpreters' perceptions of how they represent the identities of deaf people, and the role of trustworthiness in relation to mediation and representation of identities. Discussion of the key themes of representation, relationships, ability and boundaries reveal that the indexes of trustworthiness highlighted by Jones and Sin (2013) can be applied to interpreting and the interpreter's role in ensuring that deaf signers are 'known', but that there are particular subtleties in identifying who is the trustor and who is the trustee.

The extent to which interpreters explicitly understand and consciously think about representation has opened up an interesting area of new practice considerations. How should interpreters be educated and professionally prepared to understand this aspect of their role? However, there is an ambiguous border crossing between professional responsibility for good representation of the person and inappropriate exercise of control and responsibility 'for' the deaf person. 
We would like to acknowledge some limitations of the study. We recognise that the data corpus in the form of two focus groups is a small sample, so although the groups produced similar discussions, it would have been interesting to conduct more focus groups in different parts of the country to see if the themes were consistent, or if they were influenced by local contexts in different ways. ${ }^{3}$ We believe that conducting more groups would give further insight into this topic. In hindsight, we also recognise that it would have been beneficial to have given the SLIs the opportunity to reflect on the discussion and whether/how they benefited from sharing their views and experiences. This may be relevant for purposes of education or professional training, and will be taken into consideration for any future studies.

This is an area of practice that would benefit from further exploratory research with deaf people and with other language minority groups as well as professional interpreting communities. Further work here would also be add to our understanding of how perceptions of representation may impact on wellbeing for minority language users and interpreters.

\section{Notes}

1. The Translating the Deaf Self project was funded through an Arts and Humanities Council Translating Cultures Theme Research and Innovation Grant (Ref: AH/M003426/1).

2. See https://www.qsrinternational.com/nvivo/what-is-nvivo

3. We have presented these results to several conference audiences of sign language interpreters, and as audience members have commented on how much they can relate to the findings we feel that the comments from these seven interpreters are representative.

\section{About the authors}

Jemina Napier is Professor and Chair of Intercultural Communication and Director of the Centre for Translation \& Interpreting Studies in Scotland (CTISS) at Heriot-Watt University in Edinburgh. She is a qualified sign language interpreter in British and Australian Sign Languages, and in International Sign. She is a Fellow of the Association of Sign Language Interpreters UK and the Chartered Institute of Linguists UK, a Visiting Professor at Trinity College Dublin Centre for Deaf Studies and a member of the World Federation of the Deaf Expert Group on Accessibility. Address for correspondence: Department of Languages and Intercultural Studies, Heriot-Watt University, Edinburgh, EN14 4AS, UK. Email: j.napier@hw.ac.uk 
Robert Skinner is a Scottish Graduate School of Arts and Humanities (SGSAH) funded $\mathrm{PhD}$ student in the Centre for Translation \& Interpreting Studies in Scotland (CTISS) at Heriot-Watt University in Edinburgh. He is a qualified British Sign Language interpreter and is a Fellow of the Association of Sign Language Interpreters UK. He has worked as a research assistant with CTISS on several different projects. Address for correspondence: Department of Languages and Intercultural Studies, Heriot-Watt University, Edinburgh, EN14 4AS, UK. Email: ras3@hw.ac.uk

Alys Young is Professor of Social Work Education and Research within the School of Health Sciences at the University of Manchester (where she is also the director of the Social Research with Deaf People programme) and Visiting Professor at the Centre for Deaf Studies, University of the Witwatersrand, South Africa. She is a Fellow of the Academy of Social Sciences (FAcSS). Address for correspondence: Division of Nursing, Midwifery and Social Work, School of Health Sciences, Faculty of Biology, Medicine and Health, the University of Manchester, Manchester, M13 9PL. Email: alys.young@manchester.ac.uk

Rosemary Oram is an Economic and Social Research Council (ESRC) funded $\mathrm{PhD}$ student in the Social Research with Deaf People (SORD) programme in the Division of Nursing, Midwifery and Social Work at the University of Manchester. She is a deaf qualified social worker and has worked as a research assistant with the SORD programme on several different projects. Address for correspondence: Division of Nursing, Midwifery and Social Work, School of Health Sciences, Faculty of Biology, Medicine and Health, The University of Manchester, Manchester, M13 9PL. Email: rosemary.oram@postgrad.manchester.ac.uk

\section{References}

Bat-Chava, Yael (2000) Diversity of deaf identities. American Annals of the Deaf 145 (5): 420-427. https://doi.org/10.1353/aad.2012.0176

Batterbury, Sarah, Paddy Ladd and Mike Gulliver (2007) Sign language peoples as indigenous minorities: Implications for research and policy. Environment and Planning 39 (12): 2899-2915. https://doi.org/10.1068/a388

Boutin, Daniel and Keith Wilson (2009) Professional jobs and hearing loss: A comparison of deaf and hard of hearing consumers. Journal of Rehabilitation 75 (1): 36-40.

Bucholtz, Mary and Kira Hall (2004) Language and identity. In Alessandro Duranti (ed.) A Companion to Linguistic Anthropology, 369-394. Malden, MA: Blackwell. https://doi. org/10.1002/9780470996522.ch16

Burr, Vivien (2015) Social Constructionism (3rd edition). London: Routledge.

Candlin, Christopher. N. and Johnathan Crichton (eds) (2013a) Discourses of Trust. London: Palgrave Macmillan. 
Candlin, Christopher. N. and Johnathan Crichton (2013b) From ontology to methodology: Exploring the discursive landscape of trust. In Christopher N. Candlin and Johnathan Crichton (eds) Discourses of Trust, 1-20. Basingstoke, UK: Palgrave Macmillan. https://doi. org/10.1007/978-1-137-29556-9_1

De Clerck, Goedele (2017) Deaf Epistemologies, Identity and Learning: A Comparative Perspective. Washington, DC: Gallaudet University Press.

De Meulder, Maartje (2016) The Power of Language Policy: The Legal Recognition of Sign Languages and the Aspirations of Deaf Communities. Unpublished doctoral dissertation, University of Jyväskylä, Jyväskylä, Finland.

De Wit, Maya and Irma Sluis (2014) Sign language interpreter quality: The perspective of deaf sign language users in the Netherlands. The Interpreter's Newsletter 19: 63-85.

Dickinson, Jules (2014) Sign Language Interpreting in the Workplace. Coleford, UK: Douglas McLean.

Edwards, Rosalind (2013) Power and trust: An academic researcher's perspective on working with interpreters as gatekeepers. International Journal of Social Research Methodology 16 (6): 503-514. https://doi.org/10.1080/13645579.2013.823276

Edwards, Rosalind, Claire Alexander and Bogusia Temple (2006) Interpreting trust: Abstract and personal trust for people who need interpreters to access services. Sociological Research Online 11 (1). https://doi.org/10.5153/sro.1077

Edwards, Rosalind, Bogusia Temple and Claire Alexander (2005) Users' experiences of interpreters: The critical role of trust. Interpreting 7 (1): 77-96. https://doi.org/10.1075/ intp.7.1.05edw

Feyne, Stephanie (2015) Typology of interpreter-mediated discourse that affects perceptions of the identity of Deaf professionals. In Brenda Nicodemus and Keith Cagle (eds) Signed Language Interpretation and Translation Research: Selected Papers from the First International Symposium, 49-70. Washington, DC: Gallaudet University Press.

Feyne, Stephanie (2018) Variation in perceptions of the identity of interpreted Deaf lecturers. In Christopher Stone and Lorraine Leeson (eds) Interpreting and the Politics of Recognition, 119-137. London: Routledge. https://doi.org/10.4324/9781315619224-7

Gambetta, Diego (1988) Can we trust trust? In Diego Gambetta (ed.) Trust: Making and Breaking Cooperative Relations, 213-237. Oxford: Basil Blackwell.

Gillespie, Alex (2008) Trust in everyday interaction. In Ivana Marková and Alex Gillespie (eds) Trust and Distrust: Sociocultural Perspectives, 121-130. Charlotte, NC: Information Age Publishing.

Haug, Tobias, Karen Bontempo, Lorraine Leeson, Jemina Napier, Brenda Nicodemus, Beppie Van den Bogaerde and Myriam Vermeerbergen (2017) Deaf leaders' strategies for working with signed language interpreters: An examination across seven countries. Across Languages and Cultures 18 (1): 107-131. https://doi.org/10.1556/084.2017.18.1.5

Holcomb, Thomas (2018) Going beyond trust: Protecting my integrity as a Deaf academic. In Thomas Holcomb and David Smith (eds) Deaf Eyes on Interpreting, 162-173. Washington, DC: Gallaudet University Press. 
Howarth, Caroline (2016) Representations, identity and resistance in communication. In Derek Hook, Bradley Franks and Martin W. Bauer (eds) The Social Psychology of Communication (2nd edition), 153-168. Basingstoke, UK: Palgrave Macmillan.

Hsieh, Elaine, Hyejung Ju and Haiying Kong (2010) Dimensions of trust: The tensions and challenges in provider-interpreter trust. Qualitative Health Research 20 (2): 170-181. https://doi.org/10.1177/1049732309349935

Ibarra, Herminia (1999) Provisional selves: Experimenting with image and identity in professional adaptation. Administrative Science Quarterly 44 (4): 764-791. https://doi.org/ $10.2307 / 2667055$

Jackson, Heather (2013) Talking and doing trust in community relations. In Christopher N. Candlin and Johnathan Crichton (eds) Discourses of Trust, 315-329. Basingstoke, UK: Palgrave Macmillan. https://doi.org/10.1007/978-1-137-29556-9_20

Joseph, John E. (2004) Language and Identity: National, Ethnic, Religious. Basingstoke, UK: Palgrave Macmillan.

Leigh, Irene (2009) A Lens on Deaf Identities. Oxford: Oxford University Press.

Marková, Ivana, Per Linell and Alex Gillespie (2008) Trust and distrust in society. In Ivana Marková and Alex Gillespie (eds) Trust and Distrust: Sociocultural Perspectives, 3-28. Charlotte, NC: Information Age Publishing.

Marra, Meredith and Jo Angouri (2011) Investigating the negotiation of identity: A view from the field of workplace discourse. In Jo Angouri and Meredith Marra (eds) Constructing Identities at Work, 1-16. Basingstoke, UK: Palgrave. https://doi. org/10.1057/9780230360051_1

Mayer, Roger C., Davis, James H. Davis and David F. Schoorman (1995) An integrative model of organisational trust. Academy of Management Review 20 (3): 709-734. https:// doi.org/10.5465/amr.1995.9508080335

Miner, Annette (2017) Professional roles and responsibilities in designated interpreting. In Marta Biagini, Michael S. Boy and Claudia Monacelli (eds) The Changing Role of the Interpreter: Contextualising Norms, Ethics and Quality Standards, 77-101. New York: Routledge. https://doi.org/10.4324/9781315621531-5

Napier, Jemina (2011) 'It's not what they say but the way they say it.' A content analysis of interpreter and consumer perceptions of signed language interpreting in Australia. International Journal of the Sociology of Language 207: 59-87. https://doi.org/10.1515/ ijsl.2011.003

Napier, Jemina and Lorraine Leeson (2016) Sign Language in Action. Basingstoke, UK: Palgrave Macmillan.

Napier, Jemina and Meg Rohan (2007) An invitation to dance: Deaf consumers' perceptions of signed language interpreters and interpreting. In Melanie Metzger and Earl Fleetwood (eds) Translation, Sociolinguistic, and Consumer Issues in Interpreting, 159-203. Washington, DC: Gallaudet University Press.

Napier, Jemina, Alys Young, Rosemary Oram and Robert Skinner (2019) 'When I speak people look at me': British deaf signers' use of bimodal translanguaging strategies and the 
representation of identities. Journal of Translation and Translanguaging in Multilingual Contexts 5 (2): 95-120. https://doi.org/10.1075/ttmc.00027.nap

Sarangi, Srikant (2010) Reconfiguring self/identity/status/role: The case of professional role performance in healthcare encounters. Journal of Applied Linguistics and Professional Practice 7 (1): 79-100. https://doi.org/10.3726/978-3-0351-0122-5/5

Sin, Samantha and Alan Jones (2013) Achieving professional trustworthiness: Communicative expertise and identity work in professional accounting practice. In Christopher N. Candlin and Johnathan Crichton (eds) Discourses of Trust, 151-165. Basingstoke, UK: Palgrave Macmillan. https://doi.org/10.1007/978-1-137-29556-9_10

Slay, Holly and Delmonize Smith (2011) Professional identity construction: Using narrative to understand the negotiation of professional and stigmatized cultural identities. Human Relations 64 (1): 85-107. https://doi.org/10.1177/0018726710384290

Tipton, Rebecca (2010) On trust: Relationships of trust in interpreter-mediated social work encounters. In Mona Baker, Maeve Olohan and María Calzada Pérez (eds) Text and Context: Essays on Translation and Interpreting in Honour of Ian Mason, 188-208. Manchester: St Jerome. https://doi.org/10.4324/9781315759739

Van Langenhove, Luk and Ron Harré (1994) Cultural stereotypes and positioning theory. Journal of Theory and Social Behaviour 24 (4): 359-372. https://doi.org/10.1111/ j.1468-5914.1994.tb00260.x

Weber, Linda and Allison Carter (2003) The Social Construction of Trust. New York: Springer.

Young, Alys, Rosemary Oram and Jemina Napier (2019) Hearing people perceiving deaf people through sign language interpreters at work: On the loss of self through interpreted communication. Journal of Applied Communication Research 47 (1): 90-110. https://doi. org/10.1080/00909882.2019.1574018

Young, Alys, Jemina Napier and Rosemary Oram (in press) The translated deaf self, ontological (in)security and deaf culture. The Translator. 